\title{
Phase diagram of interacting pion matter and isospin charge fluctuations
}

\author{
O. S. Stashko, ${ }^{1}$ O. V. Savchuk, ${ }^{2}$ R. V. Poberezhnyuk $\odot,{ }^{3}$ V. Vovchenko $\odot,{ }^{4}$ and M. I. Gorenstein $\oplus^{3}$ \\ ${ }^{1}$ Taras Shevchenko National University of Kyiv, 03022 Kyiv, Ukraine \\ ${ }^{2}$ Frankfurt Institute for Advanced Studies, Giersch Science Center, D-60438 Frankfurt am Main, Germany \\ ${ }^{3}$ Bogolyubov Institute for Theoretical Physics, 03680 Kyiv, Ukraine \\ ${ }^{4}$ Nuclear Science Division, Lawrence Berkeley National Laboratory, Berkeley, California 94720, USA
}

(Received 11 February 2021; revised 29 April 2021; accepted 1 June 2021; published 11 June 2021)

\begin{abstract}
Equation of state and electric (isospin) charge fluctuations are studied for matter composed of interacting pions. The pion matter is described by self interacting scalar fields via a $\phi^{4}-\phi^{6}$-type Lagrangian. The mean-field approximation is used, and interaction parameters are fixed by fitting lattice QCD results on the isospin density as a function of the isospin chemical potential at zero temperature. Two scenarios for fixing the model parameterswith and without the first-order phase transition — are considered, both yielding a satisfactory description of the lattice data. Thermodynamic functions and isospin charge fluctuations are studied and systematically compared for these two scenarios, yielding qualitative differences in the behavior of isospin charge susceptibilities. These differences can be probed by lattice simulations at temperatures $T \lesssim 100 \mathrm{MeV}$.
\end{abstract}

DOI: 10.1103/PhysRevC.103.065201

\section{INTRODUCTION}

The Bose-Einstein condensation (BEC) $[1,2]$ is a fascinating phenomenon that occurs in a system of bosons when a macroscopic amount of particles occupies the zeromomentum state. This century-old phenomenon, observed experimentally in cold atomic gases [3-6], is predicted to occur in very different physical systems, ranging from condensed-matter physics to high-energy nuclear physics, astrophysics, and cosmology (see, e.g., Refs. [7-15]). A theoretical description of the BEC appears to be rather sensitive to delicate details of particle interactions [16-25].

In the present work we study the BEC phenomenon in strongly interacting QCD matter. The effective low-energy degrees of freedom in QCD are pions-the three pseudoGoldstone bosons in the confined phase. The pions obey the Bose-Einstein statistics, thus an emergence of the BEC of pions is possible and has been predicted to occur at large isospin chemical potentials, both in effective QCD theories $[26,27]$ and in first-principle lattice QCD simulations [28,29]. In nature, the pion BEC may occur during the cooling of the early Universe [30], in the gravitationally bound pion stars $[29,31,32]$, or as a nonequilibrium phenomenon in heavy-ion collisions $[8,9,33]$. The hypothetical boson stars [34-36] may exist and can be a candidate for the dark matter in the Universe [37-42].

Published by the American Physical Society under the terms of the Creative Commons Attribution 4.0 International license. Further distribution of this work must maintain attribution to the author(s) and the published article's title, journal citation, and DOI. Funded by $S C O A P^{3}$.
Different effective QCD descriptions of the phase diagram of interacting pion matter with a BEC include chiral perturbation theory [43,44], the Nambu-Jona-Lasinio model [45,46], the linear sigma model $[47,48]$, the Polyakov-loop extended quark meson model $[49,50]$, functional renormalization group [51,52], hard thermal loops [53], etc. Recently, a possibility of the BEC in the pion system at zero chemical potential was considered within a Skyrme-like model including both attractive and repulsive interaction terms [14,54,55]. Effects of repulsive interactions on the BEC of pions were studied in Ref. [56] at nonzero chemical potential. The system of pions at zero chemical potential was described [14] by an effective Lagrangian with the attractive $\left(\phi^{4}\right)$ and repulsive $\left(\phi^{6}\right)$ terms of a scalar field $\phi$. In the present paper we extend this model to the finite isospin ${ }^{1}$ chemical potential $\mu_{I}$.

The phase diagram on the whole plane of isospin chemical potential $\mu_{I}$ and temperature $T$ is investigated. Most macroscopic systems with both repulsive and attractive interactions between constituents display the first-order liquid-gas phase transition (FOPT) which is ended by the critical point (CP). Therefore, these phenomena can also be expected for the interacting pions in addition to the BEC.

Lattice QCD results support an existence of the pion BEC at finite isospin chemical potential [28]. We use the recent lattice data at zero temperature to fix the repulsive and attractive interaction parameters of the model. Then, thermodynamic functions and electric (isospin) charge fluctuations up to the fourth order are calculated in the $\left(\mu_{I}, T\right)$ plane. Two different scenarios are employed and systematically compared. The first one includes only the repulsive interactions via

\footnotetext{
${ }^{1}$ We use the common simplified terminology and call the third component of isospin (electric charge) the isospin charge.
} 
the $\phi^{6}$ term, but not the attractive $\phi^{4}$ term. In this case no FOPT transition is observed, only the BEC transition. The second possibility takes into account both the repulsive and attractive pion-pion interactions. In this case the FOPT is observed at small $T$ and generates a nontrivial interplay between the FOPT and BEC transitions on the phase diagram. The measures of the isospin charge fluctuations - scaled variance, skewness, and kurtosis - appear to be very sensitive to a presence of the $\mathrm{CP}$ and $\mathrm{BEC}$ phenomena. They are used to differentiate these two scenarios.

The paper is organized as follows: the theoretical description of interacting pion system is presented in Sec. II. The two choices of the interaction potential, the fixing of the model parameters, and the resulting phase diagrams are discussed. Section III is dedicated to fluctuations of the isospin charge, in particular the scaled variance, skewness, and kurtosis are discussed in some detail. The summary in Sec. IV closes the paper.

\section{INTERACTING-PION SYSTEM}

\section{A. Model formulation}

The three pions species, $\left(\pi^{+}, \pi^{-}, \pi^{0}\right)$, are represented as a triplet of interacting pseudoscalar fields $\phi=\left(\phi_{1}, \phi_{2}, \phi_{3}\right)$ that are described by an effective relativistic Lagrangian density:

$$
\mathcal{L}=\frac{1}{2}\left(\partial_{\mu} \phi \partial^{\mu} \phi-m_{\pi}^{2} \phi^{2}\right)+\mathcal{L}_{\text {int }}\left(\phi^{2}\right),
$$

where $m_{\pi}$ is the vacuum pion mass ${ }^{2}$ and $\mathcal{L}_{\text {int }}$ is the interaction part of the Lagrangian. We omit here the electromagnetic interactions. Consider now this system in statistical equilibrium within the grand canonical ensemble (GCE). The independent variables are the temperature $T$ and the isospin chemical potential $\mu_{I}$. The isospin chemical potential couples to the conserved isospin charge, the pion species $\pi^{+}, \pi^{-}$, and $\pi^{0}$ carry the isospin charges of $+1,-1$, and 0 , respectively.

To proceed, we apply a relativistic mean-field approximation, i.e., series $\mathcal{L}_{\text {int }}$ in terms of $\delta \sigma=\phi^{2}-\sigma$, where $\sigma=\left\langle\phi^{2}\right\rangle$ is the expectation value of the scalar field and $\langle\ldots\rangle$ denotes the GCE averaging. The effective mean-field Lagrangian can then be represented as [14]

$$
\mathcal{L} \approx \frac{1}{2}\left[\partial_{\mu} \phi \partial^{\mu} \phi-M^{2}(\sigma) \phi^{2}\right]+p_{\mathrm{ex}}(\sigma),
$$

where $M(\sigma)$ is the effective pion mass and $p_{\mathrm{ex}}(\sigma)$ is the socalled excess pressure,

$$
M^{2}(\sigma)=m_{\pi}^{2}-2 \frac{d \mathcal{L}_{\text {int }}}{d \sigma}, \quad p_{\text {ex }}(\sigma)=\mathcal{L}_{\text {int }}-\sigma \frac{d \mathcal{L}_{\text {int }}}{d \sigma} .
$$

The effective Lagrangian form of Eq. (2) implies that the main effect of interactions in our description leads to an appearance of a medium-dependent effective mass $M(\sigma)$. The excess pressure $p_{\mathrm{ex}}(\sigma)$ - the second term on the right-hand side of Eq. (2)-ensures the proper counting of the interaction energy.

The details of the model formulation can be found in Refs. [14,57]. This model was previously used to describe the pion system at zero chemical potential [14] and the system of interacting alpha particles [57]. In the present study we

\footnotetext{
${ }^{2}$ We use the natural units $\hbar=c=k=1$ and assume equal masses of all three pion species, $m_{\pi}=140 \mathrm{MeV}$.
}

apply this model to the new physical situation and consider the pion system at non-negative values of the isospin chemical potential $\mu_{I} \geqslant 0$. The results at $\mu_{I} \leqslant 0$ can then be obtained by interchanging $\pi^{+}$and $\pi^{-}$. Values of $\mu_{I}>0$ correspond to positive values of the isospin charge density $n_{I} \equiv n_{+}-n_{-}>$ 0 , where $n_{+}$and $n_{-}$correspond to $\pi^{+}$and $\pi^{-}$particle number densities, respectively. We consider the possible BEC of the positively charged pions in this regime. The expectation value of the scalar field $\sigma$ is presented as (see Refs. [14,57] for the derivation details)

$$
\sigma\left(T, \mu_{I}, M\right)=\sum_{i} \sigma_{i}^{\mathrm{th}}\left(T, \mu_{i}, M\right)+\sigma_{+}^{\mathrm{bc}},
$$

where $\sigma_{i}^{\text {th }}$ correspond to the contributions of the thermal pions, $i=(+,-, 0)$,

$$
\sigma_{i}^{\text {th }}\left(T, \mu_{i}, M\right)=\int \frac{d^{3} k}{(2 \pi)^{3}} \frac{n_{\mathrm{k}}\left(T, \mu_{i}, M\right)}{\sqrt{k^{2}+M^{2}}},
$$

while $\sigma_{+}^{\mathrm{bc}}$ corresponds to a possible contribution of the Bose condensate $(\mathrm{BC})$ of $\pi^{+}$. Here $\mu_{+}=\mu_{I}, \mu_{-}=-\mu_{I}, \mu_{0}=0$, and

$$
n_{\mathrm{k}}\left(T, \mu_{i}, M\right)=\left[\exp \left(\frac{\sqrt{k^{2}+M^{2}}-\mu_{i}}{T}\right)-1\right]^{-1} .
$$

We use a Skyrme-like parametrization of the interaction term:

$$
\mathcal{L}_{\text {int }}(\sigma)=\frac{a}{4} \sigma^{2}-\frac{b}{6} \sigma^{3}, \quad a \geqslant 0, \quad b>0 .
$$

In Eq. (7), $a \geqslant 0$ and $b>0$ are model parameters which define the strength of, respectively, attractive and repulsive interactions between particles. The effective mass and the excess pressure for this choice of the interaction terms are equal to

$$
\begin{gathered}
M(\sigma)=\sqrt{m_{\pi}^{2}-a \sigma+b \sigma^{2}}, \\
p_{\text {ex }}(\sigma)=-\frac{a}{4} \sigma^{2}+\frac{b}{3} \sigma^{3} .
\end{gathered}
$$

Inverting Eq. (8) with respect to $\sigma$ we obtain ${ }^{3}$

$$
\sigma=\frac{a+\sqrt{a^{2}+4 b\left(M^{2}-m_{\pi}^{2}\right)}}{2 b} .
$$

At given $T$ and $\mu_{I}$ we use the system of self-consistent equations (4) and (8) to determine $\sigma$ and $M$.

The pressure $p$, the number densities of thermal pions, $n_{i}^{\text {th }}$, and the isospin charge density $n_{I}$ can be calculated as

$$
\begin{aligned}
p=\sum_{i} \int \frac{d^{3} k}{(2 \pi)^{3}} & \frac{k^{2}}{\sqrt{k^{2}+M^{2}}} n_{\mathrm{k}}\left(T, \mu_{i}, M\right)+p_{\mathrm{ex}}(\sigma), \\
n_{i}^{\mathrm{th}} & =\int \frac{d^{3} k}{(2 \pi)^{3}} n_{\mathrm{k}}\left(T, \mu_{i}, M\right) \\
n_{I} & =\left(\frac{\partial p}{\partial \mu_{I}}\right)_{T}=n_{+}-n_{-}
\end{aligned}
$$

\footnotetext{
${ }^{3}$ The second root of Eq. (8) corresponds to mechanically unstable states.
} 
Here $n_{i}$ are the total number densities of pions. The $n_{+}$density may include a contribution from a Bose condensate (BC). The condensation does not occur if $\mu_{I}<M$. In the case $\mu_{I}<M$ only the thermal pions contribute to the total number densities,

$$
n_{+}=n_{+}^{\text {th }}, \quad n_{-}=n_{-}^{\text {th }}, \quad n_{0}=n_{0}^{\text {th }}, \quad \mu_{I}<M .
$$

The BEC of $\pi^{+}$occurs when their chemical potential $\mu_{+} \equiv$ $\mu_{I}$ reaches the value of the effective mass, ${ }^{4}$ i.e., $\mu_{I}=M$. In this case the number density $n_{+}$may receive a contribution $n_{+}^{\mathrm{bc}}$ from the BC:

$$
n_{+}=n_{+}^{\mathrm{th}}+n_{+}^{\mathrm{bc}}, \quad \mu_{I}=M .
$$

The number of densities of the two other pions species are unchanged: $n_{-}=n_{-}^{\text {th }}, n_{0}=n_{0}^{\text {th }}$. The number density of $\pi^{+}$in a condensate reads

$$
n_{+}^{\mathrm{bc}}=\mu_{I} \sigma_{+}^{\mathrm{bc}}=\mu_{I}\left(\sigma-\sum_{i} \sigma_{i}^{\mathrm{th}}\right) .
$$

In Eq. (16) the quantities $\sigma$ and $\sigma_{i}^{\text {th }}$ are calculated according to Eqs. (10) and (5), respectively.

An onset of the BEC takes place when $\mu_{I}$ reaches the effective mass $M$. This condition defines a line in the phase diagram - the BEC line. This line is calculated by substituting $M \rightarrow \mu_{I}$ and $\sigma_{+}^{\mathrm{bc}} \rightarrow 0$ into the system of equations (4), (8), and (12) and solving it with respect to $\mu_{I}$ at given value of $T$.

\section{B. Fixing the parameters using lattice data at zero temperature}

Lattice QCD simulations at finite isospin density provide constraints on the equation of state from first principles [28]. In particular, the isospin density $n_{I}\left(T=0, \mu_{I}\right)$ at zero temperature has been presented in Ref. [29]. The lattice QCD simulations of Ref. [28] were performed with inclusion of a pion source to trigger the explicit breaking of $U_{I}(1)$ symmetry and to stabilize the numerical simulations. The extrapolation is then performed to the limit of vanishing source. Just after this procedure, the lattice results are used to constrain the $a$ and $b$ interaction parameters in our model, because the model implicitly assumes a vanishing pion source. The refitting of the lattice data in the presence of the nonzero pion source term can be interesting and will almost certainly require modifications of the Lagrangian to achieve the presence of nonzero condensate across the entire phase diagram.

In the limit of zero temperature, $T=0$, the thermal pion excitations are absent, i.e., all thermal densities (12) vanish. In this case the system consists solely of the BC of $\pi^{+}$mesons, thus, the isospin density coincides with the number density of the condensed pions, $n_{I}\left(T=0, \mu_{I}\right)=n_{+}^{\mathrm{bc}}$, and the total pressure equals the excess pressure, $p\left(T=0, \mu_{I}\right)=p_{\text {ex }}$. The explicit expression for $n_{I}\left(T=0, \mu_{I}\right)$ in the considered model

\footnotetext{
${ }^{4}$ Note that chemical potential values $\mu_{I}>M$ exceeding the effective mass are forbidden because they would lead to negative occupancy numbers $n_{\mathrm{k}}(6)$ for some $k$ states.
}

follows from Eqs. (5), (10), and (16):

$$
n_{I}\left(T=0, \mu_{I}\right)=\mu_{I}\left(\frac{a+\sqrt{a^{2}+4 b\left(\mu_{I}^{2}-m_{\pi}^{2}\right)}}{2 b}\right) .
$$

\section{Scenario I: Repulsive interactions only}

In the first scenario we consider purely repulsive interactions between pions. To achieve this we set $a=0$ and $b>0 .^{5}$ Equation (17) in this case is reduced to

$$
n_{I}\left(T=0, \mu_{I}\right)=b^{-1 / 2} \mu_{I} \sqrt{\mu_{I}^{2}-m_{\pi}^{2}} \theta\left(\mu_{I}-m_{\pi}\right) .
$$

An onset of the BEC occurs at $\mu_{I}=m_{\pi}$. The isospin density is a continuous function of $\mu_{I}$ since $n_{I}\left(T=0, \mu_{I}=m_{\pi}\right)=0$. On the other hand, the $\mu_{I}$-derivative of $n_{I}$ exhibits a discontinuity at $\mu_{I}=m_{\pi}$. Therefore, the transition between vacuum and a pion-condensed phase at $\mu_{I}=m_{\pi}$ is a second-order phase transition at $T=0$. Qualitatively, this is consistent with predictions of many different theories, including, for instance, chiral perturbation theory $[26,43]$ or Polyakov-loop extended quark-meson model [49,50].

To fix the value of the parameter $b$ we fit the lattice QCD data on $n_{I}\left(T=0, \mu_{I}\right)$ of Ref. [29] in the range of chemical potentials $\mu_{I} / m_{\pi}<2$. We obtain $b \simeq 9.09 / m_{\pi}^{2}$ with $\chi^{2} /$ dof $\simeq 1.62$. A comparison with the lattice data is shown in Fig. 1(a) by the blue dashed line.

\section{Scenario II: Repulsion + attraction}

Let us turn now to the more general case when both the attractive and repulsive interactions are present, $a>0$ and $b>0$. In this case the system undergoes a first-order phase transition between vacuum (the gaseous phase) and a pioncondensed phase (the liquid phase), with the coexistence point being characterized by a vanishing pressure. To see this consider Eq. (9): this equation has two solutions for $p_{\mathrm{ex}}=p(T=$ $\left.0, \mu_{I}\right)=0$, defining the expectation values of the scalar field at the FOPT boundaries, $\sigma_{g}=0$, and $\sigma_{l}=3 a / 4 b$. This corresponds, via Eq. (8), to the following values of the effective mass in the gaseous and liquid components:

$$
M_{g}=m_{\pi}, \quad M_{l}=\sqrt{m_{\pi}^{2}-\frac{3 a^{2}}{16 b}} .
$$

The FOPT takes place at $\mu_{0}=M_{l}$. The gaseous phase at $T=0$ corresponds to the vacuum, thus, $n_{g}=0$. The isospin density jumps at $\mu_{I}=\mu_{0}$ from $n_{g}=0$ to

$$
n_{l} \equiv n_{I}\left(T=0, \mu_{I} \rightarrow \mu_{0}+0\right)=\frac{3 a}{4 b} \sqrt{m_{\pi}^{2}-\frac{3 a^{2}}{16 b}} .
$$

To fix the numerical values of $a$ and $b$ we again fit the lattice data on $n_{I}$ at $T=0$. We obtain $a \simeq 0.93$ and $b \simeq 11.39 / m_{\pi}^{2}$ with a fit quality of $\chi^{2} /$ dof $\simeq 1.35$-a slightly better fit compared with Scenario I.

\footnotetext{
${ }^{5}$ Another option would be to set $b=0$ and take $a<0$. The results in such a case are qualitatively similar to $a=0$ and $b>0$.
} 

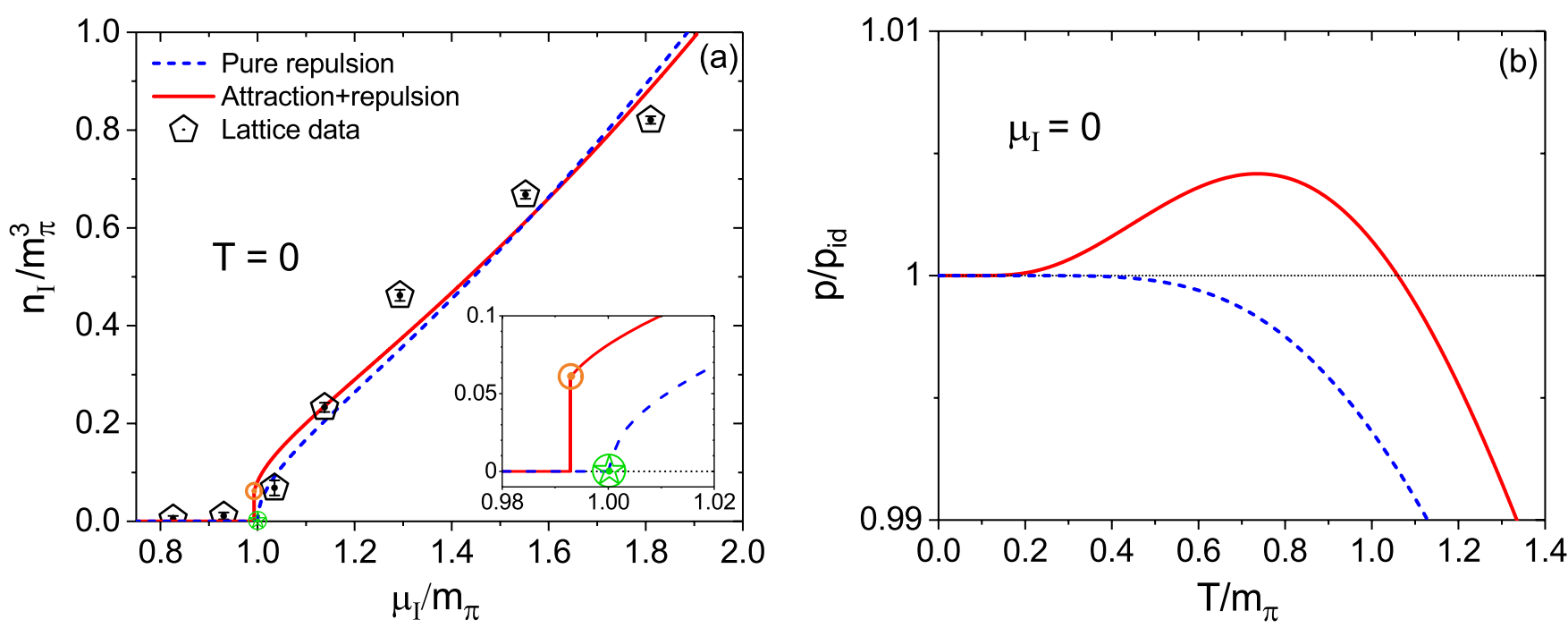

FIG. 1. (a) A comparison of the pion condensate as a function of the isospin chemical potential, $\mu_{I}$ at zero temperature, $T=0$, with lattice data of Ref. [29] is shown by solid line and dashed line for the Scenario I $(a=0)$ and Scenario II $(a>0)$, respectively. The ground state $n_{I} \approx 0.022 \mathrm{fm}^{-3}$ at $\mu_{I} \approx 0.993 m_{\pi}$ for $a>0$ and the singularity point $n_{I}=0$ at $\mu_{I}=m_{\pi}$ for $a=0$ are marked by, respectively, the red circle and the hollow green star. The inset shows the zoomed-in picture close to $\mu_{I}=m_{\pi}$. (b) The ratio of the system's pressure to the ideal-gas pressure at zero isospin chemical potential, $\mu_{I}=0$, as a function of temperature.

As discussed above, Scenario II predicts the FOPT. Using the numerical values of the $a$ and $b$ parameters fit to the lattice data, one obtains the FOPT at $\mu_{0} \approx 0.993 m_{\pi}$, where the isospin density jumps from $n_{g}=0$ to $n_{l}>0$. The values of the $\pi^{+}$density $n_{I}$ and the binding energy per particle $W$ at $T=0$ and $\mu_{I}=\mu_{0}$,

$$
\begin{gathered}
n_{I} \approx 0.060 m_{\pi}^{3} \approx 0.022 \mathrm{fm}^{-3}, \\
W \equiv \frac{\varepsilon}{n_{I}}-m_{\pi}=M_{l}-m_{\pi} \approx-1 \mathrm{MeV},
\end{gathered}
$$

obtained in Scenario II correspond to the ground state of the pion matter. This density is about seven times smaller than the normal nuclear matter density of $n_{0}=0.16 \mathrm{fm}^{-3}$, and the binding energy is about 16 times smaller than that in the nuclear ground state.

The behavior of $n_{I}$ in Scenario II at zero temperature is shown in Fig. 1(a) by the solid red line. A comparison with the lattice data and the predictions of Scenario I are also shown. Overall, the behavior of $n_{I}$ in the both scenarios is similar. Even though the nature of the phase transition differs between the two scenarios, due to the small latent heat of the FOPT in Scenario II it is difficult to distinguish it from the second-order phase transition in Scenario I using the presently available lattice data. In Sec. III we discuss fluctuations as a possibility to make such a distinction.

\section{Phase diagram at finite temperatures}

Model calculations at finite temperatures are straightforward. Important constraints on the equation of state of pion matter can be obtained at zero chemical potentials and large temperatures, $120 \leqslant T \leqslant 160 \mathrm{MeV}$, where the QCD equation of state is known from lattice QCD [58,59]. In this range, the pressure and energy density are reasonably well described by the ideal hadron-resonance gas (see, e.g., Ref. [60]). This indicates that effects of pion interactions in this regime are small.

The ideal hadron resonance gas model incorporates many mesonic resonances such as $\rho$ and $\omega$ as free particles. Counted in the hadron-resonance gas as separate hadron species, these resonances take into account an essential part of $\pi-\pi$ interactions. In the statistical model of high-energy collisions, most pions at the chemical freeze-out temperature near $T=$ $150 \mathrm{MeV}$ are not primary thermal pions but emerge after resonance decays. The role of the mesonic resonances becomes, however, less important at smaller temperature, e.g., because of their large masses, the resonance contributions to thermal equilibrium pion gas become negligible at $T<70$ $\mathrm{MeV}$ considered in the paper. Our modeling of the $\pi \pi$ interactions within the effective-mass model concerns just this hypothetical nonresonance part of the pion interactions. From a fact of the existence of atomic nuclei one concludes that the nonresonance interactions, both repulsive and attractive, exist between nucleons. It seems that straightforward experimental evidence are absent about a strength of the nonresonance repulsive and attractive forces between pions and even about their existence themselves. The lattice data at $T<70 \mathrm{MeV}$ and $\mu_{I} \approx m_{\pi}$ seem to give us a unique possibility to answer this question.

In Fig. 1(b) we plot a ratio of the pressure of interacting pions to the ideal pion gas baseline (i.e., at $a=0$ and $b=0$ ) for the two scenarios. For purely repulsive interactions (Scenario I) the pressure demonstrates small suppression relative to the ideal gas. If attractive effects are included (Scenario II) the pressure at small temperatures is higher than that of the ideal gas of pions. However, at large $T$, the repulsive effects become dominant and the pion pressure is again suppressed. In both scenarios the corrections to the ideal gas pressure at $\mu_{I}=0$ are small, not exceeding $1 \%$. This is not the case at 

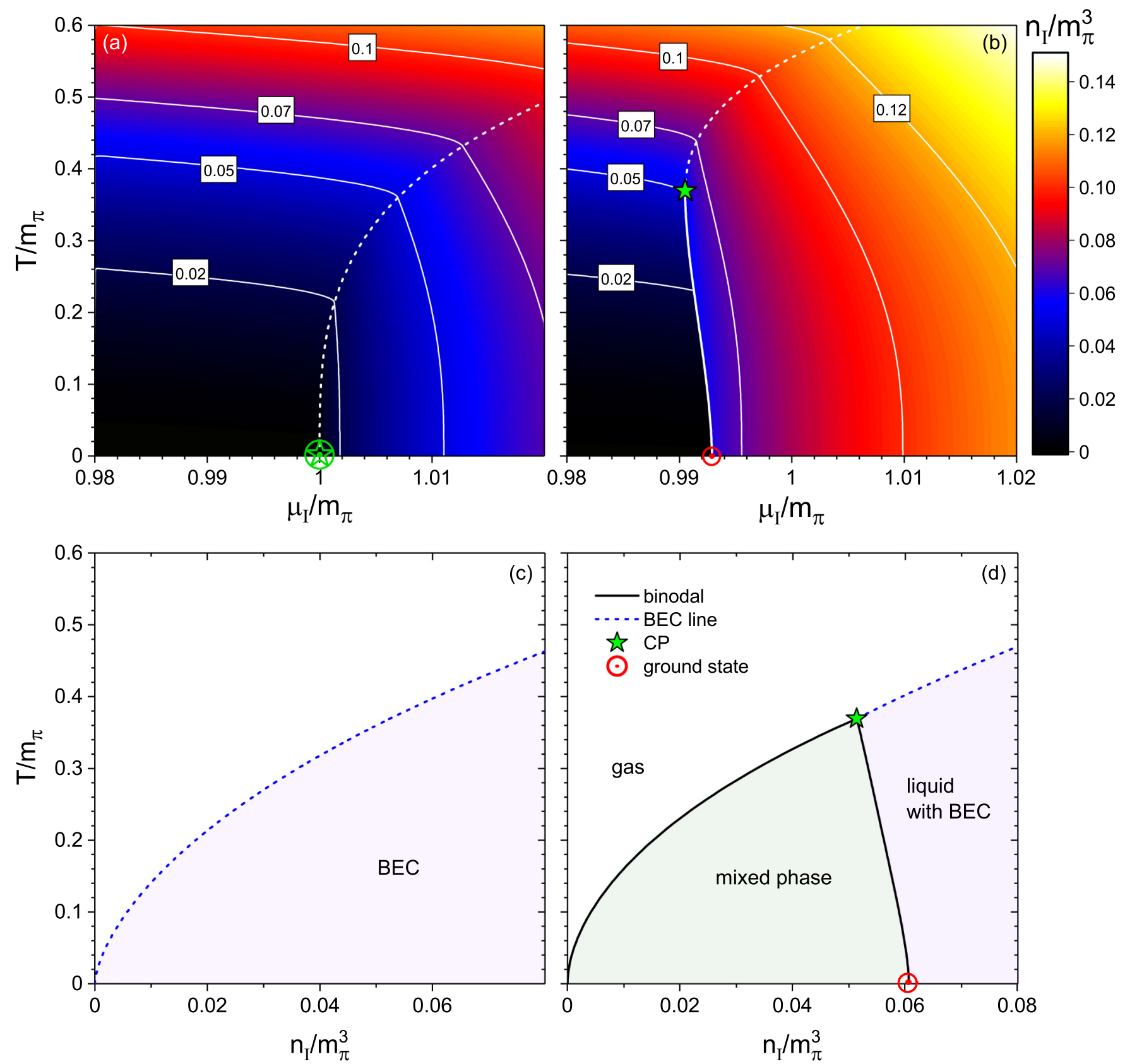

FIG. 2. Phase diagrams of the pion matter in the $\left(\mu_{I} / m_{\pi}, T / m_{\pi}\right)$ and $\left(n_{I} / m_{\pi}^{3}, T / m_{\pi}\right)$ planes, at (a), (c) $a=0$, and (b), (d) $a>0$. Dashed lines correspond to the onset of the BEC. Solid lines correspond to the first-order phase transition. The CP is marked by the green star. Coloring in panel (b) show the isospin charge density, $n_{I} / m_{\pi}^{3}$.

nonzero values of $\mu_{I}$ : the thermodynamics of the interacting pion gas differs drastically from that in the ideal pion gas in the $\left(\mu_{I}, T\right)$ region of the phase diagram where BEC pions are formed, as discussed in the following.

In Scenario I, where the attractive pion interactions are absent $(a=0)$, there is no FOPT in the pion system. An onset of the $\pi^{+}$BEC takes place when $\mu_{I}$ reaches the value of the effective mass $M$. The BEC line can thus be obtained by substituting $M \rightarrow \mu_{I}$ in the system of equations (4) and (8) and solving it with respect to $T$. The resulting BEC line $T_{\mathrm{bc}}\left(\mu_{I}\right)$ is shown in Fig. 2(a) by the dashed line. In the ideal gas limit one would obtain a vertical BEC line, $\mu_{I}=m_{\pi}$. The deviation from the ideal-gas behavior thus becomes evident as $T$ is increased. This is due to large particle number densities, and thus stronger effects of interactions, as the temperature is increased. Note that, in the ideal Bose gas, a region of the $\left(\mu_{I}, T\right)$ plane with $\mu_{I}>m_{\pi}$ is forbidden, whereas in the interacting system considered here this region is legitimate. It follows from Eq. (10) that the effective mass is always larger than the vacuum mass, $M\left(T, \mu_{I}\right)>m_{\pi}$, the pure repulsion scenario $(a=0)$. The $\left(n_{I}, T\right)$ phase diagram in the $a=0$ scenario is shown in Fig. 2(c). The thermodynamic states below the dashed lines in Figs. 2(a) and 2(c) correspond to a nonzero density of the BC, i.e., to a macroscopic 
number of $\pi^{+}$-mesons occupying the zero momentum level $k=0$.

In Scenario II, with both the repulsive $(b>0)$ and attractive $(a>0)$ pion interactions present, the FOPT phase transition takes place in addition to the BEC formation. The $\left(\mu_{I}, T\right)$ and $\left(n_{I}, T\right)$ planes are presented for this scenario in Figs. 2(b) and 2(d), respectively. The line of the FOPT is shown by a thick solid line in Fig. 2(b). This line ends in a critical point $(\mathrm{CP})$ at $T=T_{c} \approx 0.369 m_{\pi}, \mu_{I}=\mu_{c} \approx 0.991 m_{\pi}$, and $n_{I}=n_{c} \approx 0.051 m_{\pi}^{3}$, which is shown by the green star. The $\mu_{c}$ value can be expressed explicitly in terms of model parameters

$$
\mu_{c}=\sqrt{m_{\pi}^{2}-\frac{a^{2}}{4 b}} .
$$

An approximate analytical dependence of $T_{c}$ on $a, b$, and $m_{\pi}$ can also be obtained:

$$
T_{c} \approx \frac{\pi}{\sqrt{b}}\left(\frac{2 a}{\zeta(3 / 2)}\right)^{2 / 3}\left(4 b m_{\pi}^{2}-a^{2}\right)^{-1 / 6}
$$

Here $\zeta(x)$ is the Riemann zeta function. The relation (24) has been obtained by assuming $n_{-} \ll n_{0} \ll n_{+}$as well as the nonrelativistic approximation in vicinity of the CP. Using the previously obtained parameters $a$ and $b$ from fitting the lattice data one obtains $T_{c} \approx 0.393 \mathrm{~m}_{\pi}$. This is within $6 \%$ of the numerical result obtained without approximations. Note that the limit $a \rightarrow 0$ corresponds to $T_{c} \rightarrow 0$ and $\mu_{c} \rightarrow m_{\pi}$.

At the FOPT line in the $\left(\mu_{I}, T\right)$ plane the pressures of the gaseous and liquid phases are equal to each other. On the other hand, the isospin charge density $n_{I}$ has a discontinuity. The mixed phase shown in Fig. 2(d) is bounded by the gas-like (left) and liquid-like (right) binodals presented by solid lines that intersect each other at the CP. The pion states inside the mixed phase correspond to linear combinations of the diluted (gaseous) and dense (liquid) states lying on the left and right binodals, respectively. The liquid component of the mixed phase always lies below the BEC line, thus it always contains a nonzero fraction of condensed $\pi^{+}$-mesons. The gaseous component, on the other hand, does not contain the BEC.

A remarkable feature of the considered model is that the BEC line enters the mixed phase at the CP. This property of the model is robust with regard to variations in the values of the $a$ and $b$ parameters. Another peculiar property is the nonsmooth intersection of the left and right binodals at the CP.

Scenarios I and II provide a similar picture of the phase diagram at $T \gg T_{c}$. At $T \lesssim T_{c}$, on the other hand, the differences are significant. We argue that these differences can be most clearly seen by studying the behavior of isospin charge fluctuations. This is discussed in Sec. III.

The liquid-gas FOPT is a rather general phenomenon that takes place in many different physical systems, from molecular liquids to nuclear matter. This transition can be described by different mean-field models. However, all these mean-field approaches belong to the same universality class. In particular, they give the same critical exponents in a vicinity of the $\mathrm{CP}$ (see, e.g., Ref. [61]). On the other hand, properties of the BEC can look rather different in various models. In Ref. [56], several phenomenological approaches were used to describe particle repulsive effects in the Bose gas. These formulations look very similar at $\mu=0$ but appear to be drastically different in a vicinity of the BEC. A situation can become even more tangled in the system of bosons when both the repulsive and attractive forces are present, and two phenomena-the FOPT and BEC - take place simultaneously. Therefore, it is a priori not clear whether the same universality class of effective theories should lead to similar physical results. We plan to consider other types of mean-field models of the pion matter in our further studies. The present and future lattice QCD simulations will be used for these phenomenological investigations.

The QCD-inspired models such as chiral perturbation theory $[43,44]$, the Nambu-Jona-Lasinio model $[45,46]$, and the Polyakov-loop extended quark-meson model $[49,50]$ considered in the literature all appear to disfavor the existence of the FOPT. The similar conclusion comes from the linear sigma model $[47,48]$ that takes into account the (pseudo)-Goldstone nature of pions. All these models lead to a phase diagram of the pion matter without the FOPT that is qualitatively consistent with the pure repulsion scenario, namely, Scenario I considered in our paper.

However, we do believe that the ultimate conclusion should come from first-principle lattice QCD simulations. The present lattice results do not allow us to distinguish the two scenarios conclusively. In that regard, we suggest the analysis of the isospin number susceptibilities at $T<100 \mathrm{MeV}$ as a sensitive probe to distinguish the two scenarios.

\section{FLUCTUATIONS}

The two descriptions presented of the isospin charge density from the lattice results at $T=0$ both contain the BEC. Within the second description, the FOPT at $T<T_{c}$ leads to the $n_{I}$ discontinuity. We argue that the difference between the two scenarios can be probed by considering isospin charge fluctuations.

In the GCE, the $j$ th-order susceptibility of the isospin charge is determined by a $j$ th-order partial derivative of the pressure $p$ with respect to the chemical potential $\mu_{I}$ :

$$
\chi_{j}=\frac{\partial^{j}\left(p / T^{4}\right)}{\partial\left(\mu_{I} / T\right)^{j}} .
$$

First we look at the isospin charge fluctuations at $\mu_{I}=0$. The temperature dependence of the susceptibilities $\chi_{2}$ and $\chi_{4}$ at $\mu_{I}=0$ is presented in Figs. 3(a) and 3(b), respectively. At $T<m_{\pi}$ these quantities calculated for the interaction Scenarios I and II are close to those in the ideal Bose gas of pions. An increase of the fluctuations due to the attraction in Scenario II is too small and is hardly seen in Fig. 3. Thus, both scenarios are almost indistinguishable from the ideal gas at $T<m_{\pi}$. At $T>m_{\pi}$, the repulsion become significant leading to a noticeable suppression of fluctuations and the peak structures are observed. The results can be put into the context of the linear sigma model. Electric charge susceptibilities have been computed in the linear sigma model within functional renormalization-group approach in Ref. [62], and these results are qualitatively consistent with ours. Thus, both scenarios 

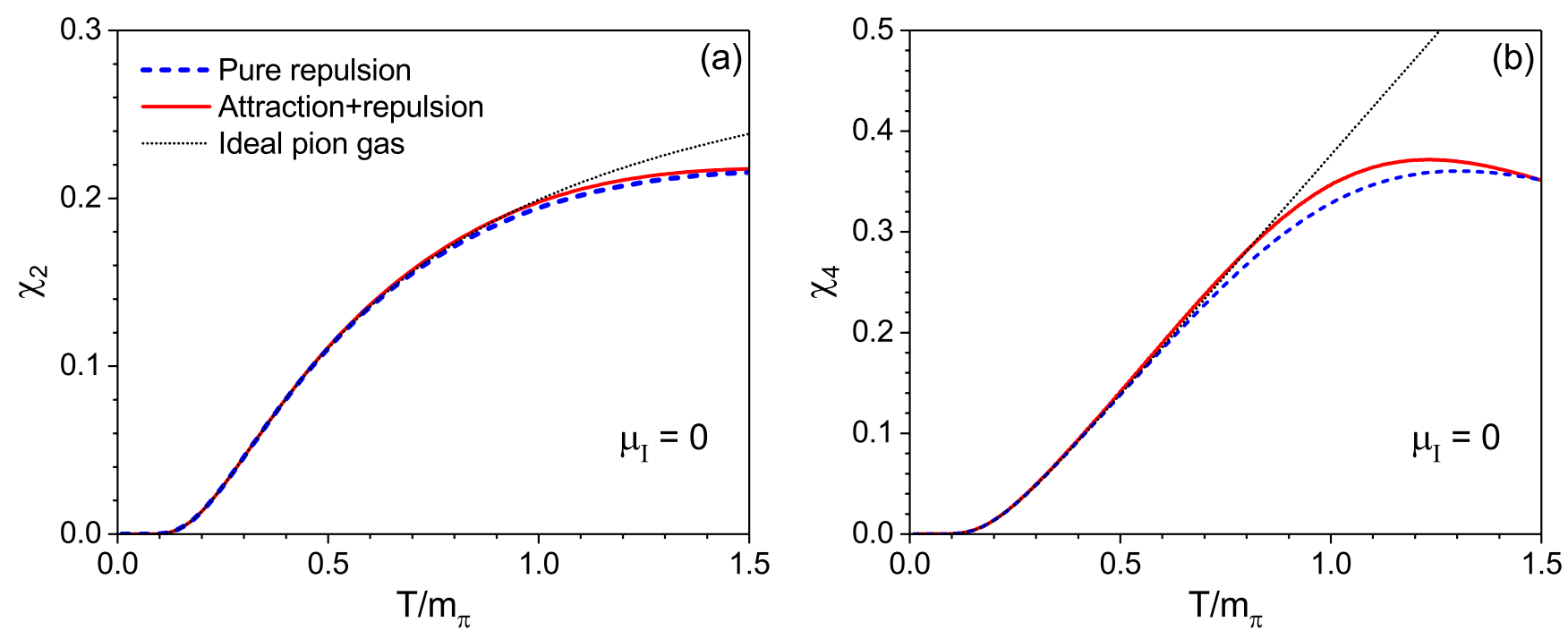

FIG. 3. Isospin fluctuation (a) $\chi_{2}$ and (b) $\chi_{4}$ as functions of temperature at $\mu_{I}=0$ for different interaction potentials.

studied here are consistent with the linear sigma model at $\mu_{I}=0$.

The susceptibilities at $\mu_{I}=0$ do not allow us to distinguish the two scenarios and determine whether the FOPT at finite isospin densities exists. Furthermore, at $T \approx m_{\pi}$ the contributions from other hadrons and resonances to the susceptibilities become important as well and also at $T \cong 155 \mathrm{MeV} \cong$ $1.15 m_{\pi}$ a crossover transition from the hadron-resonance gas to the quark-gluon plasma is expected. Thus, there appears to be a little chance to observe significant effects of the pion-pion interactions in the thermodynamic quantities at zero chemical potentials. The situation becomes very different at $\mu_{I} \approx m_{\pi}$. In this region of the phase diagram two physical phenomena become possible: BEC and FOPT. A strong increase of the electric charge fluctuations is thus expected. Rather small repulsive and attractive interactions that are barely visible at $\mu_{I}=0$ become crucially important at $\mu_{I} \approx m_{\pi}$ and lead to a qualitatively different behavior in Scenarios I and II. Thus we explore isospin charge fluctuations at finite isospin densities in the following.

Ratios of susceptibilities given by (25) can be particularly useful because such quantities are intensive in the thermodynamic limit. Some of the most well known quantities include the scaled variance $\omega$, skewness $S \sigma$, and kurtosis $\kappa \sigma^{2}$ (see, e.g., Ref. [63]):

$$
\omega=\frac{\chi_{2}}{\chi_{1}}, \quad S \sigma=\frac{\chi_{3}}{\chi_{2}}, \quad \kappa \sigma^{2}=\frac{\chi_{4}}{\chi_{2}} .
$$

Using Eq. (25) together with Eq. (11) the scaled variance $\omega$ can be written as

$$
\omega=\frac{T}{n_{I}}\left(\frac{\partial n_{I}}{\partial \mu_{I}}\right)_{T} .
$$

In the ideal pion gas the scaled variance diverges at the BEC line [8], i.e., $\omega_{\text {id }} \rightarrow \infty$ at $\mu_{I} \rightarrow m_{\pi}-0$ :

$$
\omega_{\mathrm{id}}=T^{2} \frac{m_{\pi}^{3 / 2}}{\sqrt{2} \pi n_{I}}\left(m_{\pi}-\mu_{I}\right)^{-1 / 2} \rightarrow \infty .
$$

Due to the repulsive interactions in the considered model the scaled variance remains finite. On the BEC line, $M=\mu_{I}$, one finds

$$
\omega=\frac{M T}{n_{I}}\left(\frac{\partial M}{\partial \sigma}\right)^{-1}=\frac{\mu_{I}^{2} T}{n_{I}} \frac{1}{\sqrt{b\left(\mu_{I}^{2}-\mu_{c}^{2}\right)}},
$$

where $\mu_{c}$ is given by Eq. (23). The value of $\omega$ remains also finite in a presence of the $\mathrm{BC}, n_{+}^{\mathrm{bc}}>0$. In Scenario II $(a>0), \omega$ exhibits singular behavior at the CP, $T=T_{c}$, $\mu_{I}=\mu_{c}$, where it diverges. A systematic expansion of the thermodynamic functions in a vicinity of the $\mathrm{CP}$ allows us to obtain the critical exponents. We expect that the critical exponents of the considered system are different from those in the mean-field class universality. This is due to the presence of the two order parameters, $n_{I}^{l}-n_{I}^{g}>0$ and $n_{+}^{\mathrm{bc}}>0$, which disappear simultaneously at the CP (see, e.g., Ref. [64]). A detailed discussion of this subject is, however, outside of the scope of the present study.

Scaled variance. The behavior of the scaled variance $\omega$ in the plane of temperature and isospin chemical potential is shown in Fig. 4. In Scenario I (pure repulsion), $\omega$ is a continuous function, in particular across the BEC boundary [see Fig. 4(a)]. In Scenario II (full potential), on the other hand, $\omega$ exhibits a jump discontinuity over the FOPT line and becomes divergent at the $\mathrm{CP}$ [Fig. 4(b)]. It is still a continuous function across the BEC line, however.

Note that, in the limit $a \rightarrow 0$ (Scenario I), the CP approaches $T_{c} \rightarrow 0$ and $\mu_{c} \rightarrow m_{\pi}$. Therefore, the point $\mu_{I}=$ $m_{\pi}$ at zero temperature retains some of the properties of the $\mathrm{CP}$ and exhibits large fluctuations in its vicinity. One can observe $\omega$ of any magnitude in the vicinity of this point, the exact magnitude depending on the path of approach. In particular, approaching this point along the BEC line one finds $\omega \rightarrow \infty$ at $T \rightarrow 0$.

Skewness. The skewness $S \sigma$ for Scenarios I $(a=0)$ and II $(a>0)$ is shown in Figs. 5(a) and 5(b), respectively. At small $\mu_{I} \ll m_{\pi}$, where the pion densities are small, both the pion interactions and Bose statistics effects can be neglected; 

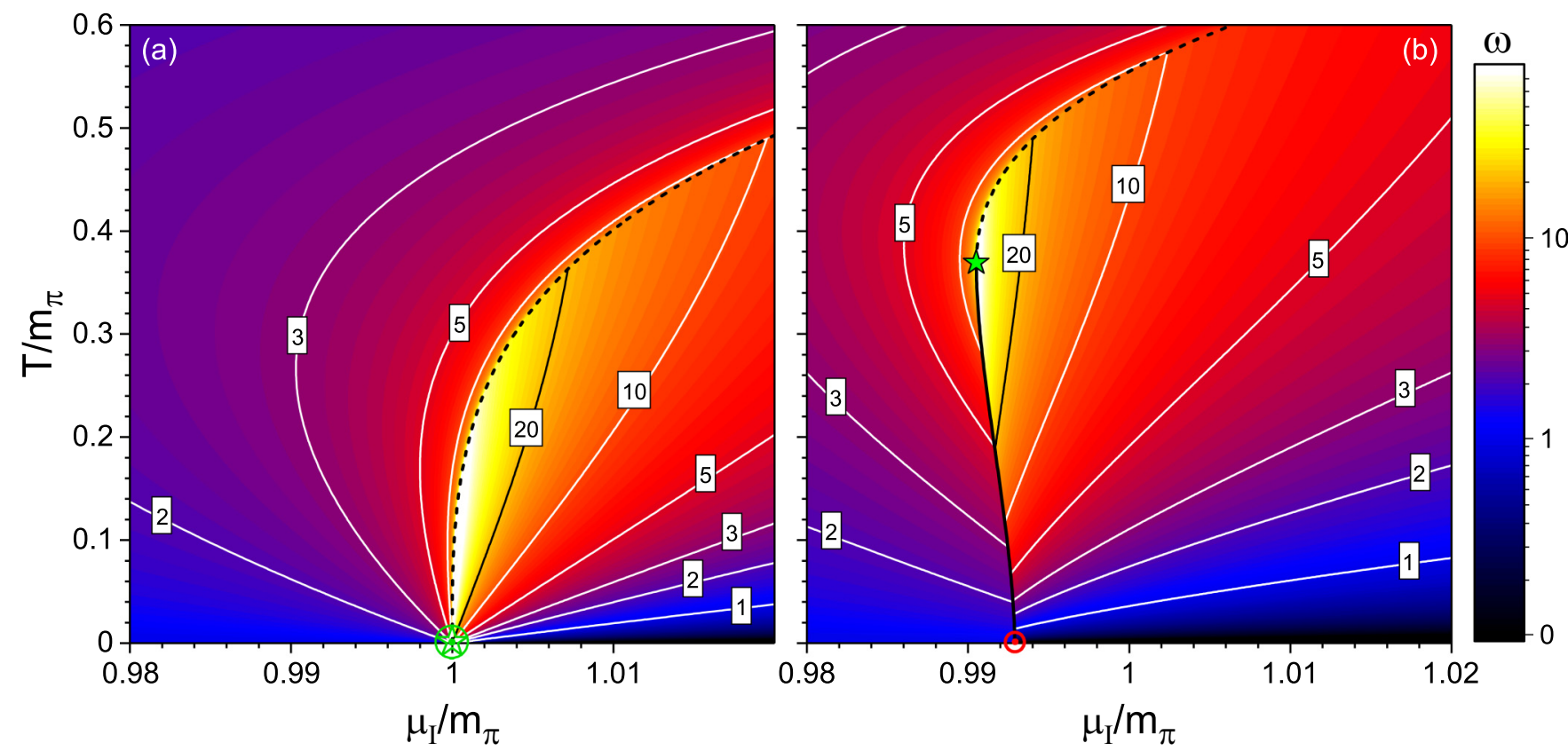

FIG. 4. The scaled variance of the isospin charge fluctuations of the pion matter in the $\left(\mu_{I} / m_{\pi}, T / m_{\pi}\right)$ plane is shown for (a) the pure repulsion case (Scenario I) and (b) the full potential case (Scenario II). Colors show values of the scaled variance. Dashed lines correspond to the onset of the BEC. Black solid lines correspond to the first-order phase transition. The CP is marked by the green star.

thus, $S \sigma \approx 1$. The skewness attains positive values in those regions of the phase diagram where there is no BC. $S \sigma$ is discontinuous along the BEC line, jumping from positive values outside the BEC phase to negative values in the phase with a BC. The above observations are valid for both scenarios. In Scenario II $(a>0) S \sigma$ shows singular behavior at the CP. The skewness can reach both $-\infty$ and $+\infty$ at the $\mathrm{CP}$ depending on the path of approach. When crossing the FOPT in Scenario II $S \sigma$ undergoes a jump discontinuity.

Kurtosis. $\kappa \sigma^{2}$ is presented in Fig. 5. In both the scenarios it also always attains positive values everywhere on the phase diagram. Kurtosis can strongly deviate from the baseline $\kappa \sigma^{2}=1$ of an ideal Boltzmann gas. This is due to the presence of interactions and Bose statistics. The largest values of the kurtosis are generally obtained in the vicinity of the BEC line. The kurtosis exhibits a nonmonotonic behavior as a function of $\mu_{I}$ at both the BEC line and the FOPT line, where it jumps down as $\mu_{I}$ is increased. $\kappa \sigma^{2}$ is an increasing function of $\mu_{I}$ elsewhere on the phase diagram. The values of $\kappa \sigma^{2}$ remain large even far away from the $\mathrm{CP}$ and the Bose condensation boundary. This is due to its large sensitivity to interactions in the system. $\kappa \sigma^{2}$ diverges at the $\mathrm{CP}$. The model does not predict negative values of $\kappa \sigma^{2}$ anywhere on the phase diagram. This is in contrast with the universal behavior of fluctuations in the Ising model $[65,66]$, as well as various model calculations [67-71], where negative values of $\kappa \sigma^{2}$ are observed in the so-called analytic crossover region above the critical temperature. In the present work the negative values of $\kappa \sigma^{2}$ are not observed because of the Bose-Einstein condensation. The BEC-line, which itself corresponds to a phase transition of a higher order, crosses the $\mathrm{CP}$, thus no region in the vicinity of the $\mathrm{CP}$ can be identified as an analytic crossover.

We would like note that $\kappa \sigma^{2}$ exhibits a singular behavior also in Scenario I $(a=0)$, at a point $\left(T=0, \mu_{I}=m_{\pi}\right)$ [see Fig. 5(c)]. Approaching this point along the BEC line, one finds $\kappa \sigma^{2} \rightarrow \infty$ at $T \rightarrow 0$.

In the present work we do not discuss the behavior of fluctuations inside the mixed phase of the FOPT. These fluctuations can be addressed by using the method developed in Ref. [72] and will be the subject of a future study.

\section{SUMMARY}

We studied the thermodynamic properties of interacting pion matter in the framework of a mean-field model with a $\phi^{4}-\phi^{6}$-type Lagrangian. The phase structure has been studied at nonzero isospin chemical potential $\mu_{I}$ that corresponds to the conserved third component of isospin. Parameters of the repulsive and attractive interactions were fixed using lattice QCD data on the isospin density as the function of the chemical potential $\mu_{I}$ at zero temperature. The lattice data can be reasonably fit within the two qualitatively different scenarios: Scenario I with only repulsive interactions, and Scenario II with both repulsive and attractive interactions. In both scenarios a phase with a Bose condensate of $\pi^{+}$pions was found to occur at sufficiently large $\mu_{I}$, the transition between ordinary pion matter and matter with a $\mathrm{BC}$ taking place along the so-called BEC lines. The presence of the attractive interactions in Scenario II leads, in addition to the $\mathrm{BEC}$, also to a first-order liquid-gas phase transition of pions with a CP at $T_{c} \approx 0.369 m_{\pi}$ and $\mu_{c} \approx 0.991 m_{\pi}$. A notable 

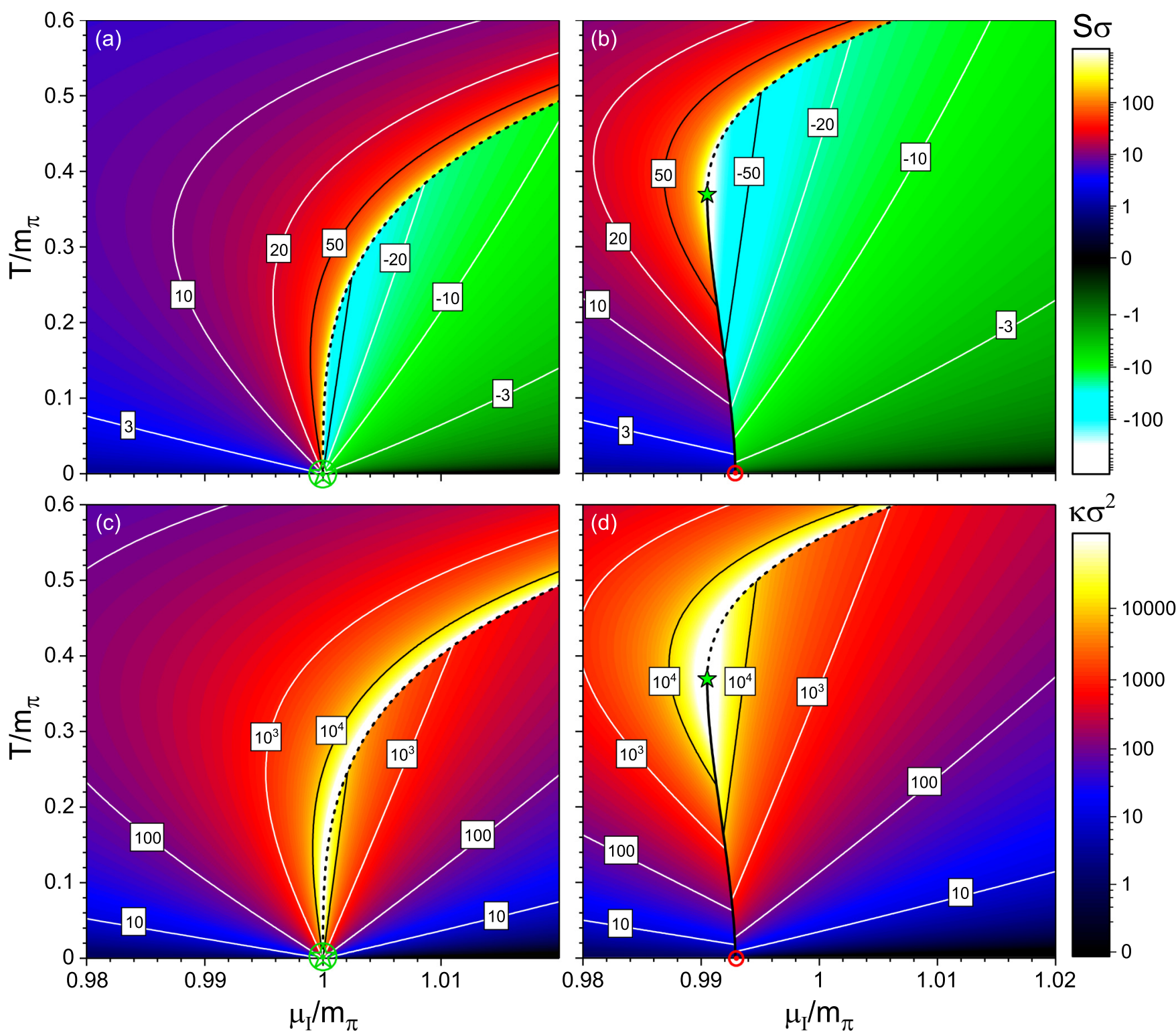

FIG. 5. (a), (b) The skewness and (c), (d) kurtosis of the isospin charge fluctuations in $\left(\mu_{I} / m_{\pi}, T / m_{\pi}\right)$ are shown for (a), (c) the pure repulsion case (Scenario I) and (b), (d) the full potential case (Scenario II).

qualitative feature of the model, present for a broad range of values of parameters $a$ and $b$, is the fact that the BEC line merges with the FOPT line at the CP. The system is characterized by two order parameters: (i) the difference $n_{l}-n_{g}>0$ between the liquid and gas phase densities corresponds to the liquid-gas transition; (ii) the density $n_{+}^{\text {bc }}$ of the Bose condensed $\pi^{+}$pions that characterizes the BEC transition. This makes the model qualitatively different from the usual systems with a CP and FOPT, where only a single order parameter is present.

The susceptibilities of isospin charge fluctuations up to the fourth order studied in the paper can serve as a robust observable to distinguish between the two different scenarios. In the both scenarios, the scaled variance $\omega=\chi_{2} / \chi_{1}$, skewness $S \sigma=\chi_{3} / \chi_{2}$, and kurtosis $\kappa \sigma^{2}=\chi_{4} / \chi_{2}$ remain finite on the
BEC line. This happens due to the repulsive interactions in the pion system, in contrast with the ideal pion gas where these measures become infinite on the BEC line. All three fluctuation measures demonstrate anomalous properties approaching the CP: $\omega \rightarrow \infty, \kappa \sigma^{2} \rightarrow \infty$, and $S \sigma$ can reach both $+\infty$ and $-\infty$ depending on a path to the CP. Note a significance of the higher-order susceptibilities (e.g., skewness and kurtosis fluctuation measures) which are highly sensitive to a presence of the CP. In Scenario I the CP is absent. In this case the anomalous fluctuations take place in the point $T=0$ and $\mu_{I}=m_{\pi}$. Approaching this point, $\omega$ and $\kappa \sigma^{2}$ can reach any value from 0 to $\infty$, and $S \sigma$ can reach any value between $-\infty$ and $+\infty$ depending on a path to the $\left(\mu_{I}=m_{\pi}, T=0\right)$ point. The susceptibilities $\chi_{i}$ can be computed in lattice QCD, which are free of the sign problem at finite $\mu_{I}$. Analysis of their behavior 
can be used to establish a point (region) of anomalously large fluctuations. Determining whether this point corresponds to zero or finite temperatures will allow us to distinguish between Scenarios I $(T=0)$ and II $\left(T=T_{c}>0\right)$. Besides, there is a qualitative difference in the behavior of the scaled variance $\omega$ near $T=0$ in Scenario I and near $T=T_{c}$ in Scenario II: $\omega \rightarrow \infty$ at $T \rightarrow T_{c}$ in Scenario II, and $\omega$ can reach any value from 0 to $\infty$ depending on the path of approaching to $T=0$ in Scenario I.

The results obtained in this paper can be used in systems where the pion densities are large and a BC of pions may occur. This can happen, for example, in heavy-ion or proton-proton collisions where the pion condensation may occur as a chemical nonequilibrium effect. Other possibilities include pion stars as well as the Early Universe which may have passed through a pion-condensed phase if the lepton flavor asymmetries during its evolution were large.

\section{ACKNOWLEDGMENTS}

We are grateful to D.V. Anchishkin, I.N. Mishustin, L.M. Satarov, and H. Stoecker for fruitful discussions. This work is supported by the Target Program of Fundamental Research of the Department of Physics and Astronomy of the National Academy of Sciences of Ukraine (N 0120U100857). The work of O.S.St. was partially supported by the $\mathrm{Na}$ tional Research Foundation of Ukraine under Project No. 2020.02/0073. V.V. acknowledges the support through the Feodor Lynen program of the Alexander von Humboldt foundation and the U.S. Department of Energy, Office of Science, Office of Nuclear Physics, under contract number DE-AC0205CH11231231.
[1] S. N. Bose, Eur. Phys. J. A 26, 178 (1924).

[2] A. Einstein, Sitzungsberichte der Preußischen Akademie der Wissenschaften (Berlin), Physikalisch-mathematische Klasse (1925), pp. 3-14.

[3] M. H. Anderson, J. R. Ensher, M. R. Matthews, C. E. Wieman, and E. A. Cornell, Science 269, 198 (1995).

[4] C. C. Bradley, C. A. Sackett, J. J. Tollett, and R. G. Hulet, Phys. Rev. Lett. 75, 1687 (1995).

[5] K. B. Davis, M. O. Mewes, M. R. Andrews, N. J. van Druten, D. S. Durfee, D. M. Kurn, and W. Ketterle, Phys. Rev. Lett. 75, 3969 (1995).

[6] F. Dalfovo, S. Giorgini, L. P. Pitaevskii, and S. Stringari, Rev. Mod. Phys. 71, 463 (1999).

[7] L. Satarov, M. Gorenstein, A. Motornenko, V. Vovchenko, I. Mishustin, and H. Stoecker, J. Phys. G 44, 125102 (2017).

[8] V. Begun and M. I. Gorenstein, Phys. Lett. B 653, 190 (2007).

[9] V. V. Begun and M. I. Gorenstein, Phys. Rev. C 77, 064903 (2008).

[10] G. C. Strinati, P. Pieri, G. Röpke, P. Schuck, and M. Urban, Phys. Rep. 738, 1 (2018).

[11] P. Nozieres and S. Schmitt-Rink, J. Low Temp. Phys. 59, 195 (1985).

[12] Y. Funaki, T. Yamada, H. Horiuchi, G. Röpke, P. Schuck, and A. Tohsaki, Phys. Rev. Lett. 101, 082502 (2008).

[13] P.-H. Chavanis and T. Harko, Phys. Rev. D 86, 064011 (2012).

[14] I. N. Mishustin, D. V. Anchishkin, L. M. Satarov, O. S. Stashko, and H. Stoecker, Phys. Rev. C 100, 022201(R) (2019).

[15] L. E. Padilla, J. A. Vázquez, T. Matos, and G. Germán, J. Cosmol. Astropart. Phys. 2019, 056 (2019).

[16] J. I. Kapusta and C. Gale, Finite-Temperature Field Theory: Principles and Applications, 2nd ed., Cambridge Monographs on Mathematical Physics (Cambridge University Press, Cambridge, 2006).

[17] J. O. Andersen, Rev. Mod. Phys. 76, 599 (2004).

[18] A. Griffin, D. W. Snoke, and S. Stringari, Bose-Einstein Condensation (Cambridge University Press, Cambridge, 1996).

[19] S. Watabe and Y. Ohashi, Phys. Rev. A 88, 053633 (2013).

[20] S. Watabe, Acta Phys. Pol., A 135, 1222 (2019).

[21] G. Baym, J.-P. Blaizot, M. Holzmann, F. Laloë, and D. Vautherin, Phys. Rev. Lett. 83, 1703 (1999).
[22] G. Baym, J.-P. Blaizot, and J. Zinn-Justin, Europhys. Lett. 49, 150 (2000).

[23] M. Holzmann and W. Krauth, Phys. Rev. Lett. 83, 2687 (1999).

[24] M. Holzmann, G. Baym, J.-P. Blaizot, and F. Laloe, Phys. Rev. Lett. 87, 120403 (2001).

[25] K. Huang, Phys. Rev. Lett. 83, 3770 (1999).

[26] D. T. Son and M. A. Stephanov, Phys. Rev. Lett. 86, 592 (2001).

[27] H. Abuki, T. Brauner, and H. J. Warringa, Eur. Phys. J. C 64, 123 (2009).

[28] B. B. Brandt, G. Endrődi, and S. Schmalzbauer, Phys. Rev. D 97, 054514 (2018).

[29] B. B. Brandt, G. Endrodi, E. S. Fraga, M. Hippert, J. SchaffnerBielich, and S. Schmalzbauer, Phys. Rev. D 98, 094510 (2018).

[30] V. Vovchenko, B. B. Brandt, F. Cuteri, G. Endrődi, F. Hajkarim, and J. Schaffner-Bielich, Phys. Rev. Lett. 126, 012701 (2021).

[31] M. Mannarelli, Particles 2, 411 (2019).

[32] J. O. Andersen and P. Kneschke, "Bose-Einstein condensation and pion stars," arXiv:1807.08951.

[33] V. Begun and W. Florkowski, Phys. Rev. C 91, 054909 (2015).

[34] F. E. Schunck and E. W. Mielke, Classical Quantum Gravity 20, R301 (2003).

[35] S. L. Liebling and C. Palenzuela, Living Rev. Relativ. 20, 5 (2017).

[36] E. Braaten, A. Mohapatra, and H. Zhang, Phys. Rev. Lett. 117, 121801 (2016).

[37] A. Suárez, V. H. Robles, and T. Matos, A Review on the Scalar Field/Bose-Einstein Condensate Dark Matter Model, in Accelerated Cosmic Expansion, edited by C. Moreno Gonzz, J. Madriz Aguilar, and L. Reyes Barrera, Astrophysics and Space Science Proceedings (Springer, 2014), Vol. 28, pp. 107-142.

[38] T. Bernal, V. H. Robles, and T. Matos, Mon. Not. R. Astron. Soc. 468, 3135 (2017).

[39] L. Visinelli, J. Cosmol. Astropart. Phys. 2016, 009 (2016).

[40] S. HajiSadeghi, S. Smolenski, and J. Wudka, Phys. Rev. D 99, 023514 (2019).

[41] J. Barranco and A. Bernal, Phys. Rev. D 83, 043525 (2011).

[42] A. M. Gavrilik, M. V. Khelashvili, and A. V. Nazarenko, Phys. Rev. D 102, 083510 (2020).

[43] P. Adhikari and J. O. Andersen, Phys. Lett. B 804, 135352 (2020). 
[44] P. Adhikari, J. O. Andersen, and M. A. Mojahed, Eur. Phys. J. C 81, 449 (2021).

[45] L.-y. He, M. Jin, and P.-f. Zhuang, Phys. Rev. D 71, 116001 (2005).

[46] J. O. Andersen and L. Kyllingstad, J. Phys. G 37, 015003 (2009).

[47] J. O. Andersen, Phys. Rev. D 75, 065011 (2007).

[48] J. O. Andersen and T. Brauner, Phys. Rev. D 78, 014030 (2008).

[49] P. Adhikari, J. O. Andersen, and P. Kneschke, Phys. Rev. D 98, 074016 (2018).

[50] A. Folkestad and J. O. Andersen, Phys. Rev. D 99, 054006 (2019).

[51] K. Kamikado, N. Strodthoff, L. von Smekal, and J. Wambach, Phys. Lett. B 718, 1044 (2013).

[52] E. E. Svanes and J. O. Andersen, Nucl. Phys. A 857, 16 (2011).

[53] J. O. Andersen, N. Haque, M. G. Mustafa, and M. Strickland, Phys. Rev. D 93, 054045 (2016).

[54] D. Anchishkin, I. Mishustin, and H. Stoecker, J. Phys. G 46, 035002 (2019).

[55] O. S. Stashko, D. V. Anchishkin, O. V. Savchuk, and M. I. Gorenstein, J. Phys. G: Nucl. Part. Phys. 48, 055106 (2021).

[56] O. Savchuk, Y. Bondar, O. Stashko, R. V. Poberezhnyuk, V. Vovchenko, M. I. Gorenstein, and H. Stoecker, Phys. Rev. C 102, 035202 (2020).

[57] L. M. Satarov, R. V. Poberezhnyuk, I. N. Mishustin, and H. Stoecker, Phys. Rev. C 103, 024301 (2021).
[58] S. Borsanyi, Z. Fodor, C. Hoelbling, S. D. Katz, S. Krieg, and K. K. Szabo, Phys. Lett. B 370, 99 (2014).

[59] A. Bazavov et al. (HotQCD Collaboration), Phys. Rev. D 90, 094503 (2014).

[60] V. Vovchenko, D. V. Anchishkin, and M. I. Gorenstein, Phys. Rev. C 91, 024905 (2015).

[61] R. V. Poberezhnyuk, V. Vovchenko, D. V. Anchishkin, and M. I. Gorenstein, Int. J. Mod. Phys. E 26, 1750061 (2017).

[62] V. Skokov, B. Friman, and K. Redlich, Phys. Lett. B 708, 179 (2012).

[63] F. Karsch and K. Redlich, Phys. Lett. B 695, 136 (2011).

[64] I. P. Ivanov, Phys. Rev. E 79, 021116 (2009).

[65] M. A. Stephanov, Phys. Rev. Lett. 107, 052301 (2011).

[66] A. Bzdak, V. Koch, and N. Strodthoff, Phys. Rev. C 95, 054906 (2017).

[67] V. Vovchenko, D. V. Anchishkin, M. I. Gorenstein, and R. V. Poberezhnyuk, Phys. Rev. C 92, 054901 (2015).

[68] V. Vovchenko, R. V. Poberezhnyuk, D. V. Anchishkin, and M. I. Gorenstein, J. Phys. A: Math. Theor. 49, 015003 (2016).

[69] J.-W. Chen, J. Deng, H. Kohyama, and L. Labun, Phys. Rev. D 93, 034037 (2016).

[70] A. Mukherjee, J. Steinheimer, and S. Schramm, Phys. Rev. C 96, 025205 (2017).

[71] A. Motornenko, J. Steinheimer, V. Vovchenko, S. Schramm, and H. Stoecker, Phys. Rev. C 101, 034904 (2020).

[72] R. V. Poberezhnyuk, O. Savchuk, M. I. Gorenstein, V. Vovchenko, and H. Stoecker, Phys. Rev. C 103, 024912 (2021). 